\title{
Beamforming Approaches for Untethered, Ultrasonic Neural Dust Motes for Cortical Recording: a Simulation Study
}

\author{
Alexander Bertrand ${ }^{1}$, Dongjin Seo ${ }^{2}$, Filip Maksimovic ${ }^{2}$, Jose M. Carmena ${ }^{2,3}$, \\ Michel M. Maharbiz ${ }^{2}$, Elad Alon ${ }^{2}$, and Jan M. Rabaey ${ }^{2}$
}

\begin{abstract}
In this paper, we examine the use of beamforming techniques to interrogate a multitude of neural implants in a distributed, ultrasound-based intra-cortical recording platform known as Neural Dust [1]. We propose a general framework to analyze system design tradeoffs in the ultrasonic beamformer that extracts neural signals from modulated ultrasound waves that are backscattered by free-floating neural dust (ND) motes. Simulations indicate that high-resolution linearly-constrained minimum variance beamforming sufficiently suppresses interference from unselected ND motes and can be incorporated into the ND-based cortical recording system.
\end{abstract}

\section{INTRODUCTION}

Brain-machine interfaces (BMI) have potential as therapeutic technology to improve the quality of life of people suffering from debilitating neurological conditions [2], [3]. Current wireless neural implants, however, have insufficient longevity (months to a few years) and a prohibitively small number of channels (10's to 100's) [4], [5] for a viable human implant. Chronic recording from thousands of sites in a clinically relevant manner with little or no tissue response remains a challenge.

This paper explores beamforming approaches for one possible neural sensing platform based on a distributed, ultrasonic backscattering system called Neural Dust (ND) first proposed in [1]. The system, shown in Fig. 1, consists of a large number of free-floating, $<100 \mu m$ ND motes (NDMs) that measure extracellular electrophysiological potentials. The sub-dural interrogator sends an ultrasonic (US) carrier wave to targetted NDMs. The NDMs then modulate the local neural signal onto the reflected carrier so that the signals are passively communicated to the interrogator. Near-field EM communication and power transfer is used for a trans-cranial link to the external transceiver.

As an extension to [1], we examine various approaches to efficiently interrogate a multitude of NDMs using an array of interrogating transducers. We discuss the mathematical channel model of the ND system and perform a simulation study to compare the performance of two beamforming

\footnotetext{
${ }^{1}$ KU Leuven - Dept. Electrical Engineering, Stadius Center for Dynamical Systems, Signal Processing, and Data Analytics. Correspondence to: alexander.bertrand@esat.kuleuven.be.

${ }^{2}$ Department of Electrical Engineering and Computer Sciences, University of California, Berkeley.

${ }^{3}$ Helen Wills Neuroscience Institute, University of California, Berkeley.

This work was supported by a Postdoctoral Fellowship of the Research Foundation - Flanders (FWO) for A.B., the NSF Graduate Fellowship for D.S., and the Bakar Fellowship for J.M.C. and M.M.M. The authors thank N. Narevsky (UC Berkeley), P. Ledochowitsch (Allen Institute), and M. Shapiro (Caltech) for advice.
}

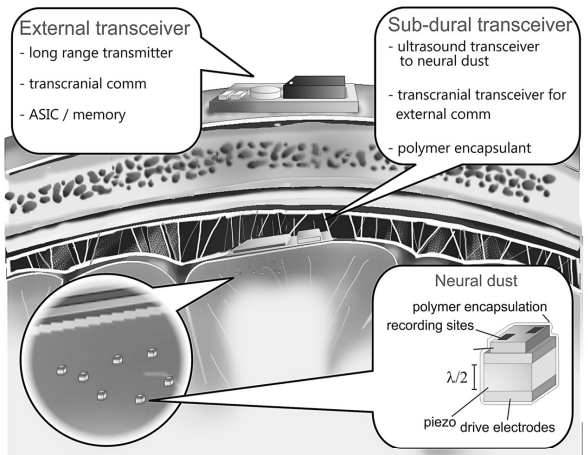

Fig. 1. Schematic illustration of the neural dust system.

(BF) techniques: delay-and-sum and linearly constrained minimum variance $\mathrm{BF}$. We investigate whether the interference from other NDMs can be sufficiently mitigated to successfully identify each NDM. However, questions related to, e.g., throughput requirements are left for future work. The paper concludes with a discussion of the challenges associated with using beamforming as part of the ND system.

\section{MATHEMATICAL FRAMEWORK}

\section{A. Channel Model}

Our model simplifies the ND system to a 1-D grid as shown in Fig. 2. A linear array of $1 \mathrm{~mm}$ interrogators, each containing multiple sub-mm US transducers (TDs) to ensure far-field operation, transmits US waves to a grid of NDMs at a depth of $2 \mathrm{~mm}$ in the neocortex [1]. The interrogators are separated by $0.1 \mathrm{~mm}$ to maximize spatial coverage and to facilitate implantation.

The US signal $S_{k q}(t)$ received at the $k$-th NDM sent by the $q$-th TD is modeled as

$$
S_{k q}(t)=H_{k q} \cdot X_{q}(t)
$$

with

$$
H_{k q}=\theta(k, q) \cdot g\left(d_{k q}\right) \cdot e^{-j \frac{2 \pi d_{k q}}{\lambda}}
$$

where $d_{k q}$ is the distance between NDM $k$ and TD $q, \lambda$ is the wavelength of the US carrier wave, $g\left(d_{k q}\right)$ is a real-valued path loss constant of US in brain tissue (typically in the range of $0.3-1.2 \mathrm{~dB} /(\mathrm{cm} \cdot \mathrm{MHz})[1]), \theta(k, q)$ is a normalized real-valued directivity gain factor based on the geometry of the TD, and $X_{q}(t)$ is the time-domain representation of the US carrier signal transmitted by TD $q$. In the following, we set $X_{q}(t)=1$, i.e., the US wave is a sinusoid, and we use a carrier frequency of $10 \mathrm{MHz}$ (in tissue, this yields $\lambda=150$ $\mu m)$, as motivated in [1]. 


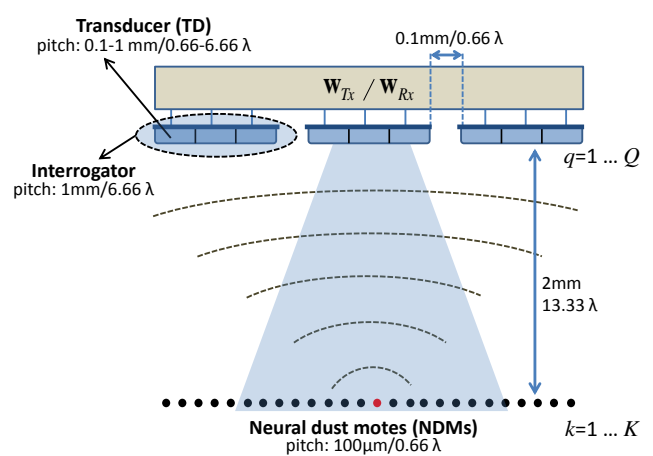

Fig. 2. 1-D simplification of the neural dust system.

The superimposed contributions from each transmitting TD at NDM $k$, represented as $S_{k}(t)=\sum_{q} S_{k q}(t)$, are modulated by the measured neural signal $V_{k}(t)$ at the $k$-th NDM and the modulated signal $V_{k}(t) \cdot S_{k}(t)$ is backscattered with an omnidirectional reflection pattern.

Let $\mathbf{V}(t)=\operatorname{Diag}\left\{V_{1}(t), \ldots, V_{K}(t)\right\}$ be a $K \times K$ diagonal matrix containing the neural signals of the different NDMs on its diagonal entries, where $K$ is the total number of NDMs. Let $R_{q}(t)$ denote the signal that TD $q$ observes when all the NDMs reflect their respective signal $V_{k}(t) \cdot S_{k}(t)$, $\forall k \in\{1, \ldots, K\}$, and define $\mathbf{r}(t)$ as the $Q$-dimensional vector where the $q$-th entry is defined as $R_{q}(t)$, where $Q$ is the total number of TDs (over all interrogators). By reciprocity, the complete transmit-receive model can then be written as

$$
\mathbf{r}(t)=\mathbf{H}^{T} \cdot \mathbf{V}(t) \cdot \mathbf{H} \cdot \mathbf{x}(t)+\mathbf{n}(t)
$$

where the $(k, q)^{t h}$ element of $\mathbf{H}$ in (3) is given by (2) and where $\mathbf{n}(t)$ represents added channel and receiver noise.

We are interested in extracting each diagonal element of $\mathbf{V}(t)$, i.e., all the neural signals of the individual NDMs. To obtain a good estimate of the neural signals, we will apply a beamforming algorithm to reduce the interference caused by the double mixing process with the matrix $\mathbf{H}$.

Note that this model does not incorporate impedance mismatches at the input and output terminal of the channel as discussed in [1]. This loss factor strongly depends on the distance between the interrogator and the NDM as shown in Fig. 3, and therefore includes an extra directional dependency in the model (2). However, unlike the model proposed in [1], which is a coupled one-to-one link between a single NDM and a single TD, the model in (3) explicitly decouples the transmit (Tx) and receive ( $R x)$ signal path, where the Tx TD and the Rx TD can be different.

It is therefore necessary to build a 3 -way tensor $\mathscr{H} \in$ $\mathbb{C}^{K \times Q \times Q}$, where the entry $H_{k, q_{1}, q_{2}}$ represents the (normalized) channel response for the signal $V_{k}(t)$ when TD $q_{1}$ is receiving and TD $q_{2}$ is transmitting. The entry $H_{k, q_{1}, q_{2}}$ is then defined as (compare with (2))

$$
H_{k, q_{1}, q_{2}}=\sqrt{\Delta P(d)} \cdot \theta\left(k, q_{1}\right) \cdot \theta\left(k, q_{2}\right) \cdot e^{-j \frac{2 \pi\left(d_{k q_{1}}+d_{k q_{2}}\right)}{\lambda}}
$$

and where $\Delta P(d)$ denotes the change in the TD input power level during a spiking event for the effective (averaged) distance of $d=\left(d_{k q_{1}}+d_{k q_{2}}\right) / 2$. The link model that generates

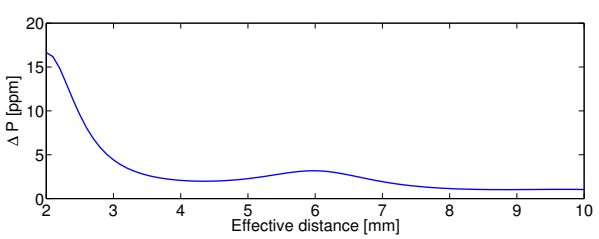

Fig. 3. Change in the TD input power level during a spiking event (in $\mathrm{ppm}$ ) as a function of the distance between the NDM and the TD [1].

the $\Delta P(d)$ curve in Fig. 3 includes a worst-case path loss of $1.2 \mathrm{~dB} /(\mathrm{cm} \cdot \mathrm{MHz})$, and therefore $g\left(d_{k q}\right)$ is not explicitly added in (4).

Unless stated otherwise, all simulation results in this paper are obtained using the more accurate tensor-based model in (4). However, for the sake of simplicity, we will use the explicit model in (3) to describe various beamforming approaches.

\section{B. Beamforming Approaches}

Beamforming is a widely-used sensor array processing technique that exploits spatial coherence between multiple sensor or transducer signals to suppress interference [6]. In the ND system, two different BF vectors $\mathbf{w}_{T x}$ and $\mathbf{w}_{R x}$ can be applied, i.e., one in transmit (Tx) and one in receive ( $\mathrm{Rx})$ mode, respectively. Depending on the communication bandwidth in the transcranial link, these BFs can be computed internally or externally. From (3), the BF output signal when interrogating NDM $k$ is defined as

$$
z_{k}(t)=\mathbf{w}_{R x}^{H} \cdot \mathbf{H}^{T} \cdot \mathbf{V}(t) \cdot \mathbf{H} \cdot \mathbf{w}_{T x}^{*} \cdot X(t)+\mathbf{w}_{R x}^{H} \cdot \mathbf{n}(t)
$$

where the superscripts $*$ and $H$ denote the complex conjugate and the complex conjugate transpose operator, respectively.

The delay-and-sum (DAS) BF [6] is a simple and computationally cheap BF technique that applies a delay or phase shift to each signal in the sensor array, equal to the corresponding phase shifts in (2), i.e.,

$$
\mathbf{w}_{T x}=\mathbf{w}_{R x}=\left[\begin{array}{c}
e^{-j \frac{2 \pi d_{k 1}}{\lambda}} \\
\vdots \\
e^{-j \frac{2 \pi d_{k Q}}{\lambda}}
\end{array}\right]
$$

such that the signals constructively interfere in the target direction. In the following, all simulations assume a DAS $\mathrm{BF}$ in the Tx mode.

For the Rx mode, an alternative approach is to use an adaptive beamforming technique called linear constrained minimum variance (LCMV) BF [6]. LCMV BF adapts its beam shape to the interference pattern using the second-order statistics of $\mathbf{r}(t)$ at the cost of computational complexity. The goal of LCMV BF is to optimize the beamformer coefficients so that the variance of the $\mathrm{BF}$ output signal is minimized while maintaining a unity gain in the steering direction, i.e.,

$$
\mathbf{w}_{\mathrm{Rx}}=\underset{\mathbf{w}}{\arg \min }\left(E\left\{\left|\mathbf{w}^{H} \mathbf{r}(t)\right|^{2}\right\}, \text { s.t. } \mathbf{w}^{H} \mathbf{h}_{k}=1\right)
$$

where $E\{\cdot\}$ denotes the expected value operator (taken over the full signal length in the simulations in this paper), and 


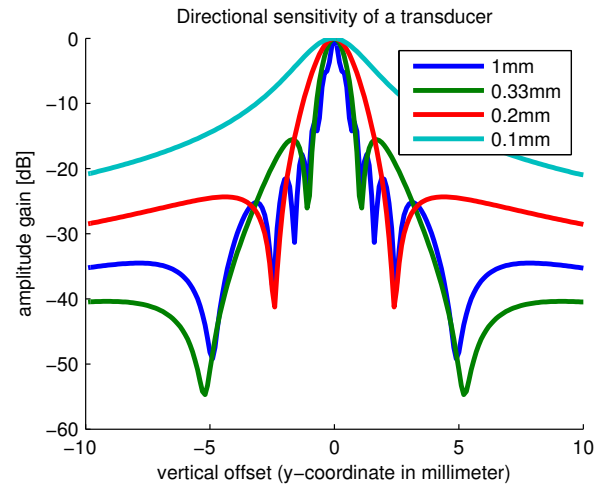

Fig. 4. Directional gain for square planar transducers of different sizes.

where the steering vector $\mathbf{h}_{k}$ contains the channel responses from the target NDM $k$ to the $Q$ TDs, i.e., the $k$-th row of H. The closed-form solution of (7) is [6]

$$
\mathbf{w}_{\mathrm{Rx}}=\frac{\mathbf{R}^{-1} \mathbf{h}_{k}}{\mathbf{h}_{k}^{H} \mathbf{R}^{-1} \mathbf{h}_{k}}
$$

where $\mathbf{R}=E\left\{\mathbf{r}(t) \cdot \mathbf{r}(t)^{H}\right\}$ is the TD signal covariance matrix (assuming $\mathbf{r}(t)$ is zero-mean).

The LCMV BF takes the interference pattern into account and removes as much interfering energy as possible. However, this also means that it is very sensitive to modeling errors in the steering vector $\mathbf{h}_{k}$. Fortunately, the sensitivity can be reduced by adding a scaled identity matrix as a regularization term to $\mathbf{R}$, to trade off steering sensitivity with interference cancellation [7] (see also Subsection III-C).

\section{Performance Metrics}

In the simulations below, the signals $V_{k}(t)$ consist of (uncorrelated) artificial spike signals with a signal-to-noise ratio (SNR) of $0 \mathrm{~dB}$, as shown in Fig. 5. Based on these signals, we define two performance metrics.

The signal-to-error ratio (SER) quantifies the total amount of noise or interference that is added by the ultrasonic interrogation process, based on the error between the $\mathrm{BF}$ output signal $z_{k}(t)$ and the signal $V_{k}(t)$ recorded at the NDM:

$$
S E R_{k}=10 \cdot \log _{10} \frac{E\left\{V_{k}(t)^{2}\right\}}{E\left\{\left(V_{k}(t)-z_{k}(t)\right)^{2}\right\}}
$$

As an alternative metric, we use a simple threshold-based spike detection algorithm with a minimum refractory period of $1.5 \mathrm{~ms}$ between two consecutive detections, and compare the misdetection rate (MDR) and false discovery rate ${ }^{1}$ (FDR) between $z_{k}(t)$ and $V_{k}(t)$. The threshold is tuned manually on the signals $V_{k}(t)$ (targeting an MDR of $<30 \%$ ).

\section{SIMULATION RESULTS}

In this section, we provide and compare the design tradeoffs and the performance of the presented BF interrogation techniques. We confine ourselves to a qualitative description of the main conclusions, rather than providing detailed simulation results.

\footnotetext{
${ }^{1} \mathrm{MDR}=\mathrm{FN} /(\mathrm{TP}+\mathrm{FN})$ and $\mathrm{FDR}=\mathrm{FP} /(\mathrm{TP}+\mathrm{FP})$ where $\mathrm{FN}, \mathrm{FP}$, and TP denote the number of false negatives, false positives and true positives, respectively.
}

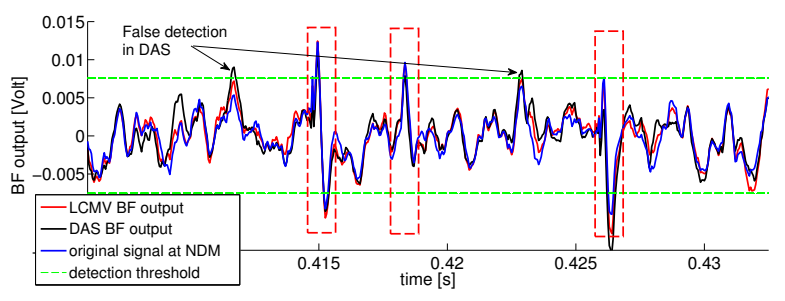

Fig. 5. BF output signal $z_{k}(t)$ compared to the original neural signal $V_{k}(t)$. Dashed boxes indicate actual spikes.

\section{A. Effect of Transducer Size}

One crucial variable in the system is the physical size of the TD elements in the $1 \mathrm{~mm}$ interrogators. The normalized directional gain $\theta$, as used in (2) is computed based on finite element modeling and is shown in Fig. 4 for varying dimension of TDs. We note that larger TDs are more directional and therefore capture less interference from neighboring NDMs, but this also limits the achievable steering angle. Unless stated otherwise, we assume $0.33 \mathrm{~mm}$-size TDs in the rest of this paper, i.e., three TDs per interrogator.

Using smaller, less directional TDs can increase the steering angle and provide more degrees of freedom in the $\mathrm{BF}$ design as the number of TDs increases. Simulations indeed show that the BF performance improves significantly when the TD size is decreased to $0.1 \mathrm{~mm}$ (see, e.g., Fig. 6 vs. Fig. 8). However, the increase in the density of TDs requires a substantially larger processing power compared to $0.33 \mathrm{~mm}$ size TDs (the LCMV processing power increases quadratically with the number of input channels). In future work, this power vs. accuracy trade-off will be further investigated.

\section{B. Comparison of beamforming configurations}

In the following, we consider a time-multiplexed interrogation process where a single NDM is interrogated in each time slot. In doing so, the interrogator closest to the target NDM can either create a Tx and/or Rx beam on its own, or multiple interrogators can cooperate by creating a joint $\mathrm{Tx}$ and/or Rx beam. Our simulations indicate that cooperation is crucial for the Rx beam (see Fig. 6). However, we observe that a single-Tx/multi-Rx configuration works better than multi-Tx/multi-Rx. This is because the interrogators are separated by more than $\lambda / 2$ and $0.33 \mathrm{~mm}$-size TDs are very directional (see Fig. 4), which makes it difficult for a neighboring interrogator to steer a sidewards $\mathrm{Tx}$ beam towards a NDM that is not in the frontal direction. This will only create more interfering backscatter from other NDMs. Although this effect vanishes for $0.1 \mathrm{~mm}$-size TDs, the single-Tx/multi-Rx configuration is never significantly outperformed by a multi-Tx/multi-Rx configuration, for an ND system with dimensions shown in Fig. 2.

For the Rx beam, we can either choose a DAS BF or an LCMV BF. As demonstrated in Fig. 7, the LCMV BF generally outperforms the DAS BF (with an SER of $6 \mathrm{~dB}$ vs. $10.3 \mathrm{~dB}$, respectively). This is because the LCMV BF optimizes its BF coefficients to minimize the amount of 


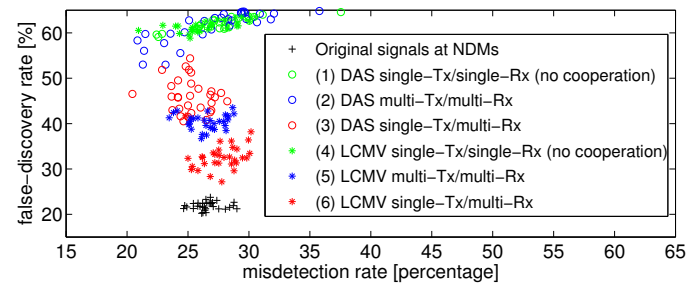

Fig. 6. Comparison between LCMV, DAS, and Tx/Rx BF config for 0.33 mm-size TDs. Different points correspond to signals from different NDMs.

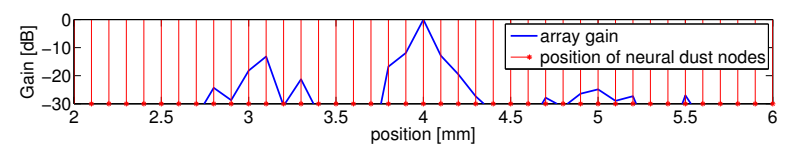

(a) $\mathrm{DAS}-\mathrm{SER}=6.06 \mathrm{~dB}, \mathrm{MDR}=27.2 \%, \mathrm{FDR}=45.8 \%$

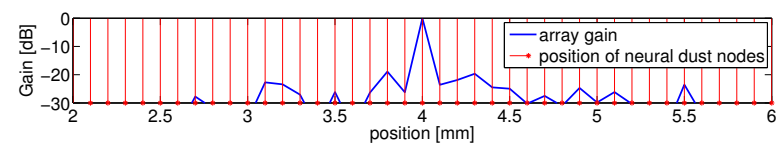

(b) LCMV - SER $=10.34 \mathrm{~dB}, \mathrm{MDR}=27.2 \%$, FDR $=32.9 \%$

Fig. 7. Beam pattern of the DAS and LCMV BF.

interference energy captured by its main beam and/or its side lobes, whereas the DAS BF cannot optimize its beam shape. This is also demonstrated in Fig. 7, which shows that the sharper beam pattern of the LCMV BF better attenuates the signals that are reflected by the non-targeted NDMs. Fig. 5 compares the BF output signals with the orignal signal $V_{k}(t)$ (in blue) as recorded at the NDM. Some false detections are noticable in the DAS BF output (this is also reflected in the MDR/FDR plot of Fig. 6).

The difference between LCMV and DAS BF becomes even more pronounced if the spacing between neighboring NDMs can be $<100 \mu m$ (simulation not shown here).

\section{Sensitivity to model mismatch}

As previously mentioned, the LCMV beamforming suffers from its high sensitivity to errors in the steering vector $\mathbf{h}_{k}$. If the LCMV BF is not exactly on-target, it may treat the target source as an interfering source and try to remove it, which could significantly degrade the performance.

Simulations show that minor uncertainty (i.e., less than $0.5 \%$ in the speed of sound in tissue) severely reduces the performance of the LCMV BF, whereas the DAS BF is less affected. This observation indicates that DAS BF is a more attractive option despite several advantages of LCMV BF. Fortunately, LCMV BF can be robustified against steering errors by applying regularization. This is demonstrated in Fig. 8 (for $0.1 \mathrm{~mm}$-size TDs). Intuitively, regularization shifts the LCMV BF coefficients closer to the DAS BF coefficients, resulting in a 'meet-in-the-middle' solution.

\section{DISCUSSION}

Our analysis demonstrates that cooperation between different interrogators is unavoidable to achieve sufficient interference suppression. The choice for hardware implementations of such BF systems is determined by the available communication bandwidth and the processing power. In order

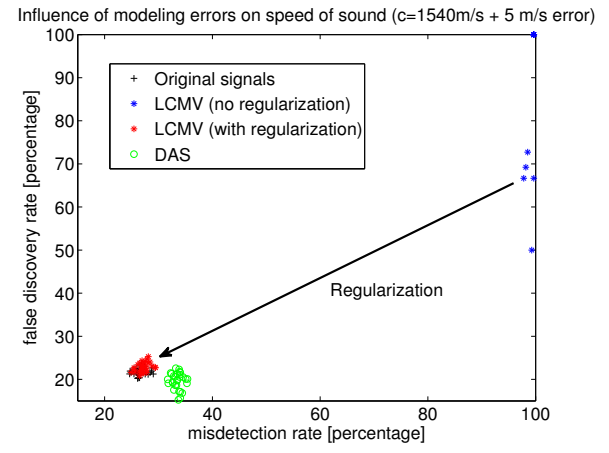

Fig. 8. Sensitivity to model mismatch of DAS and (regularized) LCMV BF for $0.1 \mathrm{~mm}$-size TDs.

to reduce the processing and communication burden, we can consider a hierarchical processing flow where a $\mathrm{BF}$ is computed in two stages, i.e., each interrogator locally computes a BF signal, which is then combined with the BF signals of other interrogators by a global 2nd-stage BF. In general, such an approach removes many degrees of freedom, and therefore the level of hierarchy should be carefully optimized. However, there also exist distributed realizations of the LCMV BF, which can be shown to be equivalent to a centralized realization, at the cost of a slower tracking [8].

Furthermore, as opposed to the current time-multiplexing method, which limits the rate of NDM interrogation, one could explore spatial multiplexing where multiple NDMs are interrogated simultaneously by the same Tx beam(s). However, it is important to consider the additional interference caused by simultaneously interrogating the NDMs.

Finally, all simulations in this paper were applied to a 1$\mathrm{D}$ grid of NDMs and interrogators, but the model can be extended to 2-D and 3-D grids. Furthermore, the degree of realism in the model can be improved by adding uncertainty in the orientation of the NDMs (i.e., random directional reflectivity patterns) and adding time-variations in the signal statistics due to micro-motion of the cortex, changes in position or orientation of the NDMs, etc. We believe that these variations are sufficiently slow to track with adaptive algorithms. However, all this remains an open challenge.

\section{REFERENCES}

[1] D. Seo, J. M. Carmena, J. M. Rabaey, E. Alon, and M. M. Maharbiz, "Neural Dust: An Ultrasonic, Low Power Solution for Chronic BrainMachine Interfaces," arXiv:1307.2196, Jul. 2013.

[2] J. L. Collinger, et al., "High-performance neuroprosthetic control by an individual with tetraplegia," The Lancet, Volume 381, Issue 9866, pp. 557-564, 2013.

[3] J. M. Carmena, "Advances in Neuroprosthetic Learning and Control," PLoS Biol vol. 11, no. 5: e1001561, 2013.

[4] T. A. Szuts, et al., "A wireless multi-channel neural amplifier for freely moving animals," Nature Neurosci, vol. 14, no. 2, pp. 263-269, 2011.

[5] W. Biederman, D. J. Yeager, N. Narevsky, A. C. Koralek, J. M. Carmena, E. Alon, J. M. Rabaey, "A Fully-Integrated, Miniaturized $\left(0.125 \mathrm{~mm}^{2}\right) 10.5 \mu \mathrm{W}$ Wireless Neural Sensor," IEEE J Solid-State Circuits, vol. 48, no. 4, pp. 960-970, Apr. 2013.

[6] B. Van Veen and K. Buckley, "Beamforming: a versatile approach to spatial filtering," IEEE ASSP Magazine, vol. 5, no. 2, pp. 4-24, 1988.

[7] R. Lorenz and S. Boyd, "Robust minimum variance beamforming," IEEE Trans Sig Proc, vol. 53, no. 5, pp. 1684-1696, 2005.

[8] A. Bertrand and M. Moonen, "Distributed LCMV beamforming in a wireless sensor network with single-channel per-node signal transmission”, IEEE Trans Sig Proc, vol. 61, no. 13, pp. 3447-3459, 2013. 